\title{
LHC-ATLAS Phase-I upgrade: calibration and simulation of a new trigger readout system for the Liquid Argon calorimeter
}

\section{G. Tateno on behalf of ATLAS LAr Calorimeter collaboration}

Department of Physics, The University of Tokyo, Hongo, Bunkyo-ku, Tokyo, Japan

E-mail: gen@icepp.s.u-tokyo.ac.jp

Aвstract: The ATLAS experiment at CERN's Large Hadron Collider is in the middle of the PhaseI upgrade for Run 3 (2021-2023) and the High-Luminosity LHC (2026-) where the instantaneous luminosities are going to be much higher than previously reached. In order to achieve a trigger rate compatible with the first stage trigger $(100 \mathrm{kHz})$ without imposing higher energy thresholds, the ATLAS Liquid Argon Calorimeter group has been working on an upgrade of its trigger readout electronics. As part of this upgrade, new trigger "Super Cells" will be introduced, increasing the granularity of the trigger readout by a factor of ten with respect to Run 2 . While the production and installation of the new electronics is underway, a calibration framework for the energy and timing measurement of the Super Cell readout is also being developed. The framework is created by using data taken in calibration runs with a pulsing system to establish the Super Cell pulse shape and other parameters, i.e. pedestal level, equivalent transverse energy for one ADC bit, and coefficients of an optimal filtering algorithm for energy calculation. In July 2019 we obtained the calibrations for the first installed digitizer board in the calorimeter end-cap. The new hardware has been verified to meet the design specifications. We are also developing a new simulation of the calorimeter using the calibrated parameters obtained during the commissioning of the new trigger readout system. The simulation takes into consideration the long bipolar pulse shape for any number of interaction per bunch crossing. This accounts for highly realistic out-of-time pileup effect.

Keywords: Calorimeters, Noble liquid detectors, Data acquisition concepts, Trigger concepts and systems, Calibration methods, Simulation methods 


\section{Contents}

1 Introduction 1

2 Upgrade of the trigger readout system 1

3 Calibration method 2

3.1 Test pulsing system 2

3.2 Calibration flow 2

4 Simulation of pileup effects with the measured pulse shapes 4

5 Summary 6

\section{Introduction}

In 2021-2023 the LHC (Large Hadron Collider) will run with the same or higher beam luminosity than in Run 2, and increases are also planned for the High-Luminosity LHC. To accommodate this higher luminosity we are upgrading the trigger readout system of the Liquid Argon (LAr) Calorimeter of the ATLAS experiment [1]. Among a number of goals, this upgrade will enhance the performance of the jet rejection. The new LAr trigger readout system uses an optimal filtering algorithm to compute the transverse energy ${ }^{1}$ and the timing of the digitized signal pulse. It has a ten times finer read out segmentation for the trigger path with respect to the Run 2 system, accomplished through so-called Super Cells. The coefficients of the optimal filtering need to be calibrated by a test pulsing system. A framework for calibration and simulation of the new trigger readout system has been developed so that we can immediately process and analyze data taken from the front-end boards which are installed on the detector during the Phase-I upgrade from 2019 to 2020.

\section{Upgrade of the trigger readout system}

Super Cells will be introduced for the trigger readout of the LAr calorimeter for the Run 3 in order to use the shower shape information of electrons and photons. In the new trigger readout system, the digitization of the Super Cell analog signal will be performed at the front-end with a sampling frequency of $40 \mathrm{MHz}$. The digitized data will be transmitted to the back-end system and be processed to precisely obtain the transverse energy and timing information of each Super Cell. There are 58 front-end crates (FECs) in total. Analog signals from the calorimeter have triangular shapes, and they are amplified and shaped with a CR-(RC) $)^{2}$ bipolar filter in the front-end boards

\footnotetext{
${ }^{1}$ ATLAS uses a spherical coordinate $\operatorname{system}(r, \theta, \phi)$ with its origin at the nominal IP in the centre of the detector and $\phi$ being the azimuthal angle around the $z$-axis along the beam pipe. The pseudorapidity is defined as $\eta=-\ln \tan (\theta / 2)$ using the polar angle $\theta$. The transverse energy is defined by $E_{\mathrm{T}}=E \sin \theta$.
} 
(FEBs) to minimize the pileup effects. ${ }^{2}$ New LAr Trigger Digitizer Boards (LTDBs) are also being installed in the frond-end crates prior to Run 3.

Transverse energy and timing are computed by the LAr Trigger prOcessing MEzzanine (LATOME) board with an optimal filtering algorithm in the back-end system. The optimal filtering algorithm provides the transverse energy of a Super Cell at the $k^{\text {th }}$ bunch crossing with the following formula:

$$
E_{\mathrm{T}}^{k}=R \sum_{i=0}^{3} a_{i}\left(S_{i}^{k}-p-b_{i}^{k}\right)
$$

where $a_{i}$ are the optimal filtering coefficients, and $p$ is the pedestal level of the Super Cell. The index $i$ specifies a sample for optimal filtering and four consecutive samples are used. ADC values $S_{i}^{k}$ and the baseline $b_{i}^{k}$ depend on the bunch crossing timing to calculate the transverse energy. ${ }^{3} R$ is the conversion factor of the computed amplitude in ADC counts to transverse energy.

Production of the new electronics (LTDBs and LATOMEs) and the installation work are under way. The front-end system must accommodate the new, additional LTDBs. Therefore, the baseplane, which transmits analog signals between boards in a crate, must be replaced. This work is the most time-consuming part. As of November 2019, the work for half the front-end crates has been done.

\section{Calibration method}

\subsection{Test pulsing system}

A simplified equivalent circuit of the test pulsing system for an individual, physical LAr cell is shown in Figure 1. The signal pulse shape from the calorimeter for a real energy deposition is triangular. The triangle pulse is received by the FEB and reaches the LTDB through the base plane. The digitized bipolar pulse on the LTDB is transmitted to the LATOME and the transverse energy is extracted from the amplitude. For calibration, test pulses with exponential shapes ${ }^{4}$ are generated by the calibration board [2] in the front-end crate. Calibration pulses are sent to the calorimeter to measure realistic pulse responses. The LATOME extracts 32 ADC samples for all 320 Super Cells and transmits them to a PC where they are recorded in a specific format. The first calibration data of the newly installed LTDB in the end-cap was obtained in July 2019.

\subsection{Calibration flow}

There are three types of calibration runs: pedestal run, delay run, and ramp run. We verified a new calibration framework using a set of data taken at the end of the autumn in 2019. The simplest calibration run is the pedestal run where no pulse is injected. For example, we measured the pedestal

\footnotetext{
${ }^{2}$ Pileup signals arise from low energy collisions in the same or neighboring bunch crossings, which are irrelevant to physics analyses.

${ }^{3}$ The so-called train structure of the LHC beam causes a baseline shift because of pileup and the characteristic of the bipolar pulse. We call the average shift from the pedestal the baseline, while the pedestal is defined as the average ADC value where no pulse is injected. The baseline is calculated taking an average of the shift at each bunch crossing timing in the train structure period.

${ }^{4} \mathrm{~A}$ triangle pulse generator could be used in the calibration board, but the complex circuit required would cause more uncertainty in the responses.
} 


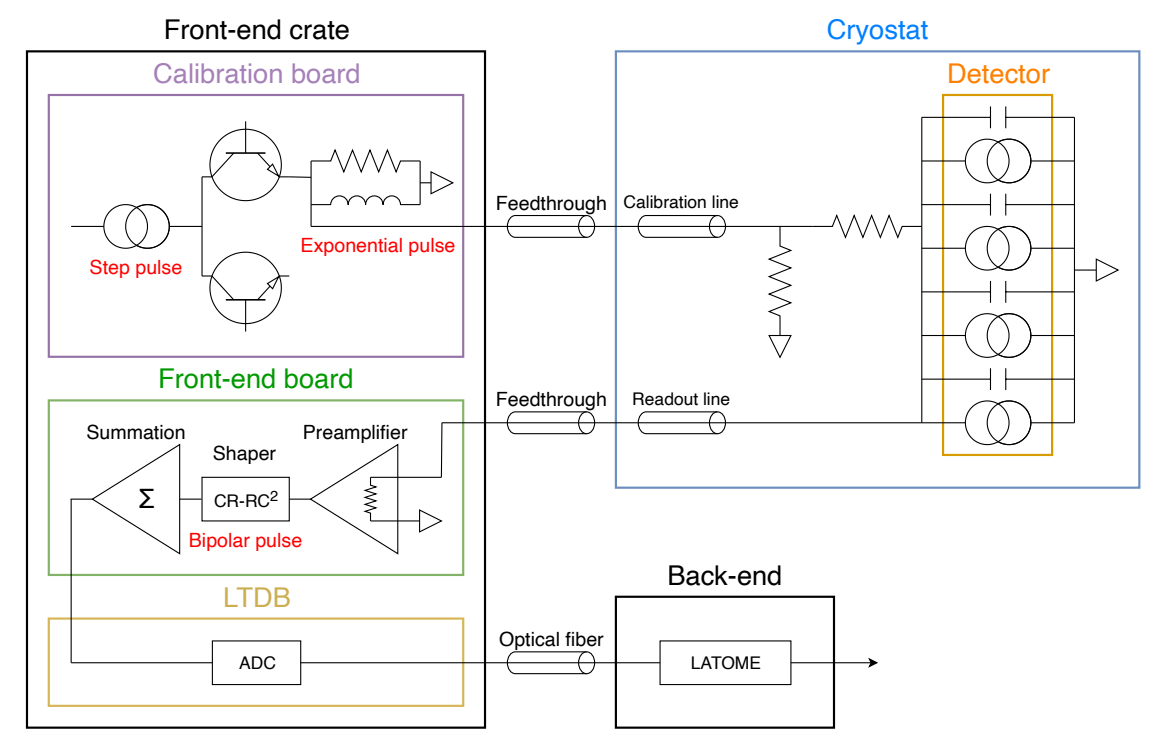

Figure 1. Simplified equivalent circuit of the test pulsing system for a LAr cell. We regard the LAr gaps as capacitors and the systems to generate triangle pulse as triangle pulse generators.

$p=915.190$ in ADC counts for a Super Cell at $(\eta, \phi)=(0.0125,-1.7211)$ in the middle layer (layer 2). The noise level and the auto-correlation are also measured for each Super Cell with the pedestal run.

Figure 2 shows the result of a delay run, in which bipolar pulses are sampled over 24 sets of pulse phases. ${ }^{5}$ By overlaying the sequences of samples for different phases accordingly, the smooth pulse shape is obtained as if it was sampled every $\frac{25}{24} \mathrm{~ns}$. The amplitude of the bipolar pulses from the LAr calorimeter is proportional to the energy. In the Super Cell discussed above, we injected $487 \mathrm{GeV}$ in transverse energy ${ }^{6}$ and observed approximately 1780 ADC counts. The transverse energy per 1 bit $\left(E_{\mathrm{T}} / \mathrm{bit}\right)$ is therefore about $274 \mathrm{MeV}$, which is consistent with the design value.

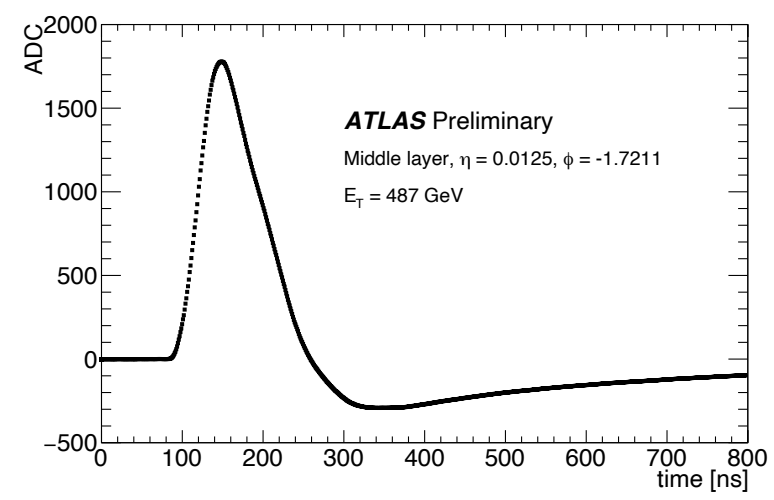

Figure 2. The calibration pulse shape of the Super Cell at $(\eta, \phi)=(0.0125,-1.7211)$ in the middle layer where the pedestal is subtracted. The average of $100 \mathrm{ADC}$ values is taken for each sample and phase. The input current corresponds to $487 \mathrm{GeV}$ in transverse energy.

\footnotetext{
${ }^{5} 100$ sets of samples are taken for each phase and averaged. The interval of phases is $25 / 24 \mathrm{~ns}$.

${ }^{6}$ The relation between input current and transverse energy was determined in a test-beam [3].
} 
The last calibration run is the ramp run, which uses 15 different energy values injected with the same phase. The pulse amplitudes are computed using an optimal filtering algorithm to compare with the injected energy. Figure 3 left shows the result of the ramp run as a relation between injected transverse energy and output ADC count. The saturation level is visible (around 600-700 GeV) as well as the $E_{\mathrm{T}} / \mathrm{bit}$, which is calculated from the linear region before saturation. In this Super Cell, $E_{\mathrm{T}} / \mathrm{bit}$ is $273.859 \mathrm{MeV}$ which is consistent with the one derived from the delay run data. Figure 3 right shows $E_{\mathrm{T}} /$ bit of each Super Cell in the $\phi=-1.7211$ region of the middle layer. The original pulse shape of the calibration run is different from the pulse shape in the physics run, as mentioned before, so the amplitude of the bipolar pulses also differs even if the same transverse energy is injected. We predict a physics pulse shape from the calibration pulse shape obtained from the delay run data by using the so-called $R T$ method [4] and correct $E_{\mathrm{T}} /$ bit. ${ }^{7}$ The ramp $R$ in Eq. (2.1) is the $E_{\mathrm{T}} /$ bit after the correction.
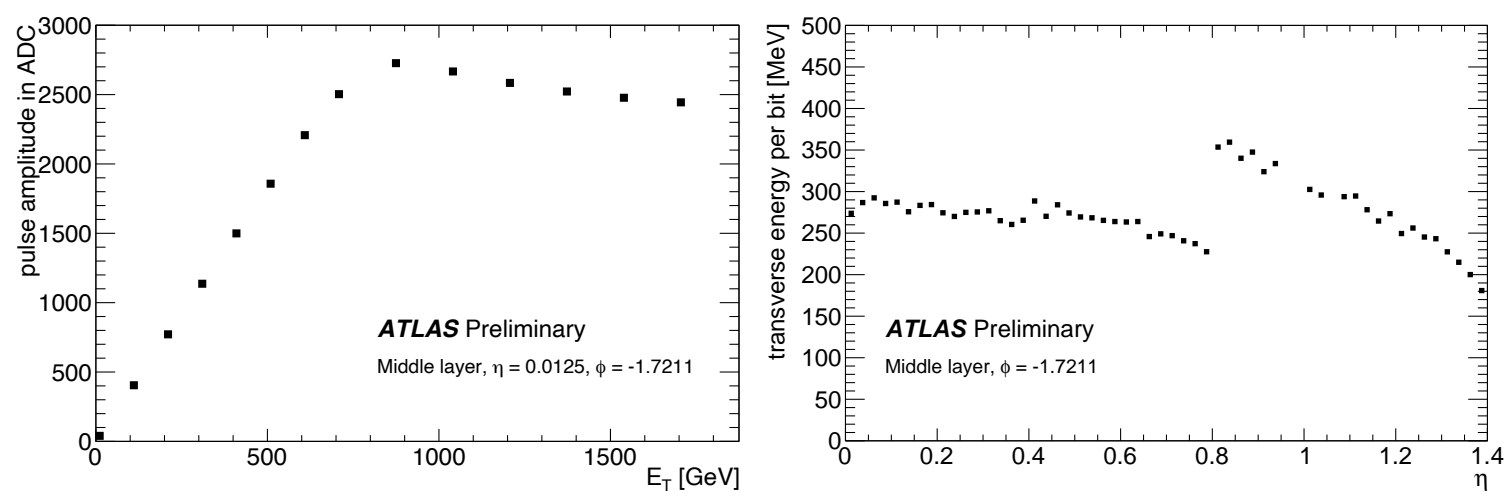

Figure 3. Input transverse energy vs output ADC value of the Super Cell at $(\eta, \phi)=(0.0125,-1.7211)$ (left) and $E_{\mathrm{T}} /$ bit of each Super Cell in the region with $\phi=-1.7211$ region (right). Both are in the middle layer. The average of $100 \mathrm{ADC}$ values is taken at each point for the left plot.

The linearity of the injected transverse energy vs the output ADC count holds even if the injected $E_{\mathrm{T}}$ exceeds $500 \mathrm{GeV}$, while the required value for a typical trigger tower is $255 \mathrm{GeV}$. Furthermore, $E_{\mathrm{T}} /$ bit in each Super Cell is consistent with the predicted value from the designed saturation level and ADC dynamic range. We have succeeded in the validation of the calibration framework of the new trigger readout system of the LAr calorimeter for the first time.

\section{Simulation of pileup effects with the measured pulse shapes}

Optimal filtering coefficients $a_{i}$ are calibrated using the auto-correlation matrix and the derived physics pulse shape. Until Run 3 starts we rely on predicted auto-correlation matrices of physics runs. We have a simple way to predict the auto-correlation using a physics pulse shape and noise information obtained in a pedestal run, but there is no guarantee that the prediction algorithm works in a high luminosity environment. For example, we might have to consider a baseline shift

\footnotetext{
${ }^{7}$ We will measure physics pulse shapes when physics runs start in 2021.
} 
caused by the bunch train structure of the LHC beam. Therefore, a simulator has been developed to investigate the optimal method to calculate the auto-correlation. ${ }^{8}$

The simulator can easily reproduce a particular bunch train structure, a given number of interactions per bunch, the pulse shape, noise, sampling phase, and so on. Figure 4 shows the input and output of the simulation. Sequences of pulses are generated by adding multiple pulses which are created by multiplying and shifting the pulse shape according to the energy and timing, respectively, and according to the given hit information for each cell. For example, users can program a sequence of high energy bipolar pulses such as in Figure 4 (a). In this simulation, the signals that we want to analyze are called events, and we produce dedicated hit information including the pileup components. In each cell, sequences of events (Figure 4 (a)), pileup (Figure 4 (b)), and electric noise (Figure 4 (c)) are summed to produce a realistic sequence (Figure 4 (d)). The zoomed plot of Figure 4 (e) clearly shows the effects of pileup and electric noise. Finally, the sequence is digitized. At this point, it is ready for analysis by the optimal filtering algorithm for the energy and timing reconstruction.
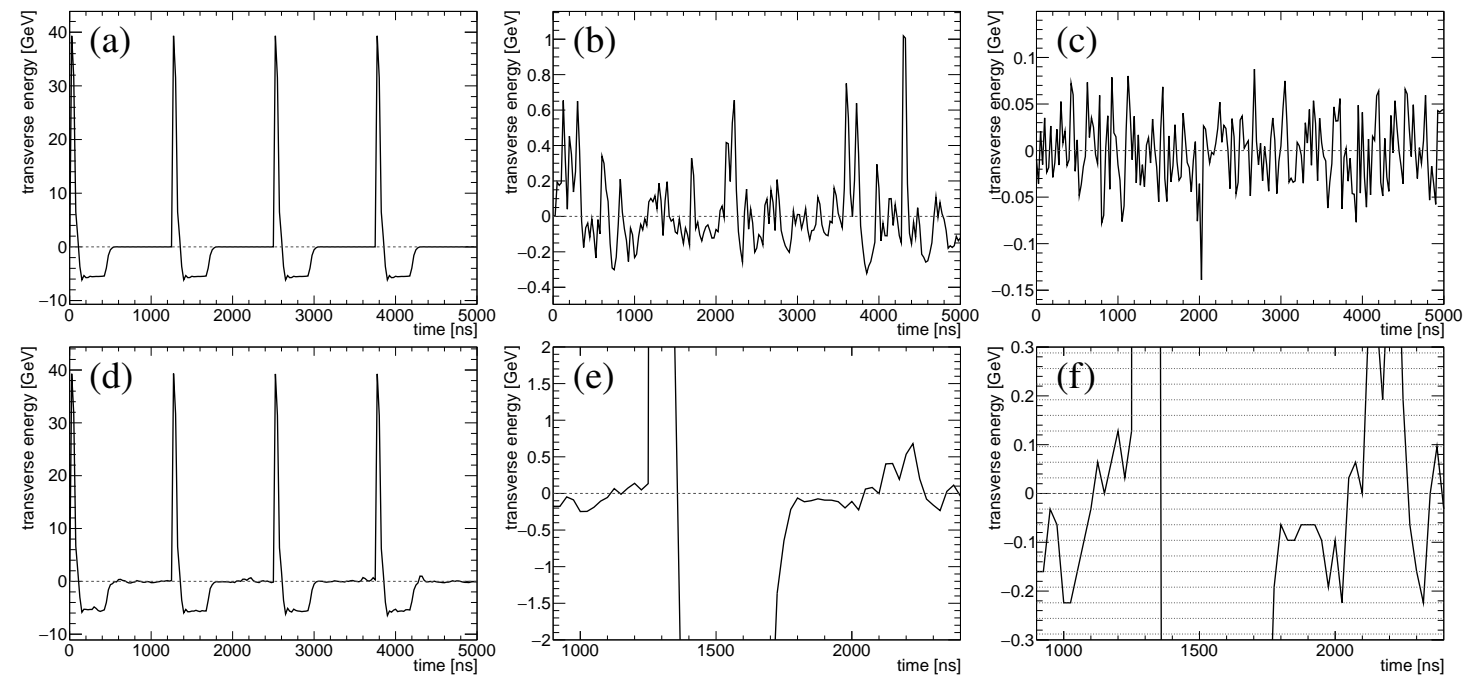

Figure 4. Output of the simplified simulator for the new trigger system. A pulse shape generated by the SPICE (Simulation Program with Integrated Circuit Emphasis) simulator is used as an example. The upper plots show a sequence of events (a), pileup (b), and electric noise (c). (d) shows the sum of these sequences. The zoomed plot is shown in (e), and the digitized output, which is multiplied by $E_{\mathrm{T}} / \mathrm{bit}$, is shown in (f).

Figure 5 shows an example of the result of the optimal filtering algorithm and selection in the simulator. The $E_{\mathrm{T}}$ output should be given only for the corresponding bunch crossing. Shifts from the ideal time, denoted as $\tau$, are the other quantity that the optimal filtering algorithm outputs. The red point is selected by the measured time phase, $|\tau|<8$ ns with $E_{\mathrm{T}}>1 \mathrm{GeV}$, which is consistent with the injected energy and timing. Now that we have an efficient pulse simulation for various bunch train structures and instantaneous luminosity conditions, the next step will be to investigate an optimal way to determine auto-correlation with the new tool.

\footnotetext{
${ }^{8}$ This simulator is new with respect to the conventional simulator, AREUS (ATLAS Readout Electronics Upgrade Simulation) [5], in that it simulates pulse shape with pileup by taking any complex train structure into account, with no limitation in the time period of pulse sequences.
} 


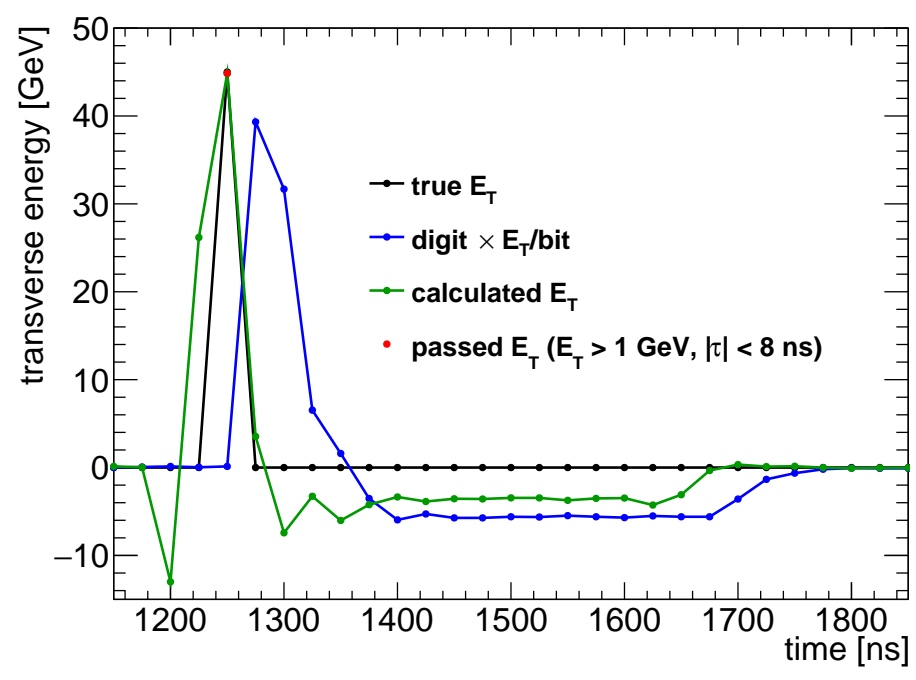

Figure 5. Example of optimal filtering and selection in the simulator for the new system of the LAr calorimeter. A pulse shape generated by the SPICE simulator is used as an example. Signals from important bunch crossings are chosen by some criteria for reconstructed energy and timing.

\section{Summary}

The new trigger readout system of the ATLAS LAr calorimeter, which is part of the Phase-I upgrade, was presented. An optimal filtering algorithm will be used to calculate the energy and timing for the Level-1 trigger. In order to calibrate the optimal filtering coefficients for each Super Cell, a calibration framework has been developed. We managed to extract the first set of coefficients using the data taken at the end of the autumn in 2019.

Optimal filtering coefficients are calibrated using the auto-correlation matrix. These calibrations may need to take into account the pileup effects with the bunch train structure of the LHC beam. Therefore, we are developing a simulator to seek an optimal way to obtain the auto-correlation matrix.

\section{References}

[1] ATLAS Collaboration, The ATLAS Experiment at the CERN Large Hadron Collider, JINST 3 (2008) S08003

[2] J. Colas, et al., Electronics calibration board for the ATLAS liquid argon calorimeters, Nucl. Instrum. Meth. A 593 (2008) 269

[3] M. Aleksa, et al., ATLAS Combined Testbeam: Computation and Validation of the Electronic Calibration Constants for the Electromagnetic Calorimeter, ATL-LARG-PUB-2006-003, https://cds.cern.ch/record/942528

[4] D. Banfi, M. Delmastro, and M. Fanti, Cell response equalisation of the ATLAS electromagnetic calorimeter without the direct knowledge of the ionisation signals, JINST 1 (2006) P08001

[5] N. Madysa, AREUS: A Software Framework for ATLAS Readout Electronics Upgrade Simulation, EPJ Web Conf. 214 (2019) 02006 